\title{
Nonlinear optics via double dark resonances
}

\author{
S. F. Yelin, ${ }^{1,2}$ V. A. Sautenkov, ${ }^{2,3}$ M. M. Kash, ${ }^{4,3}$ G. R. Welch, ${ }^{3}$ and M. D. Lukin ${ }^{5}$ \\ ${ }^{1}$ Department of Physics, University of Connecticut, Storrs, Connecticut 06269, USA \\ ${ }^{2}$ Lebedev Physics Institute, Moscow 117924, Russia \\ ${ }^{3}$ Department of Physics, Texas A\&M University, College Station, Texas 77843, USA \\ ${ }^{4}$ Department of Physics, Lake Forest College, Lake Forest, Illinois 60045, USA \\ ${ }^{5}$ Department of Physics, Harvard University, Cambridge, Massachusetts 02138, USA
}

(Received 20 June 2003; published 2 December 2003)

\begin{abstract}
Double dark resonances originate from a coherent perturbation of a system displaying electromagnetically induced transparency. We experimentally show and theoretically confirm that this leads to the possibility of extremely sharp resonances prevailing even in the presence of considerable Doppler broadening. A gas of ${ }^{87} \mathrm{Rb}$ atoms is subjected to a strong drive laser and a weak probe laser and a radio frequency field, where the magnetic coupling between the Zeeman levels leads to nonlinear generation of a comb of sidebands.
\end{abstract}

DOI: 10.1103/PhysRevA.68.063801

PACS number(s): 42.50.Gy

\section{INTRODUCTION}

Dark resonances arise from the quantum superposition states that are decoupled from coherent and dissipative interactions. They are now a well-known concept in quantum optics and laser spectroscopy, leading to concepts such as electromagnetically induced transparency (EIT) [1], lasing without inversion and increased resonant index of refraction [2], highly efficient resonant nonlinear optics [3-5], "slow" light [6-9], coherent storage of photon states [10-12], and femtosecond generation $[13,14]$.

As a rule, any perturbation of the dark state leads to undesirable decoherence. However, it was shown theoretically that "perturbing" the dark state coherently leads to multiple quantum superposition states that interact coherently. These so-called "double dark" states $[15,16]$ can be used to mitigate decoherence effects and thus enlarge the domain of dark state physics. The perturbation can come from different sources: microwave or optical coherent fields, dc fields, nonadiabatic optical coupling (i.e., effective fields). In this paper we discuss theoretically and experimentally the use of the magnetic component of a radio frequency (RF) field. We observe two sharp resonances at shifted frequencies. We also demonstrate that the double dark resonances can enhance the nonlinear optical process. In particular, we demonstrate the efficient generation of a comb of multiple frequency components associated with double dark resonances.

\section{THEORY}

\section{A. Dark resonances and electromagnetically induced transparency}

The principle of electromagnetically induced transparency (EIT) is the destructive interference of two absorption channels, which originate from a Stark-split resonance. It is based on a so-called dark resonance. For example, in a $\Lambda$ system like the one in Fig. 1(a) with the excited state $|a\rangle$ coupled to the metastable states $|b\rangle$ and $|c\rangle$ via strong driving and weak probe fields with Rabi frequencies $\Omega$ and $\alpha$, respectively, the resonant interaction Hamiltonian is $H=\hbar \Omega|c\rangle\langle a|$ $+\hbar \alpha|b\rangle\langle a|+$ H.c. The superposition state $|+\rangle \propto \Omega|c\rangle$ $+\alpha|b\rangle$ is coupled by both fields, $H=\hbar \Omega_{+}|+\rangle\langle a|+$ H.c., with $\Omega_{+}^{2}=\Omega^{2}+\alpha^{2}$, whereas the orthogonal state $|-\rangle$ $\propto \Omega|b\rangle-\alpha|c\rangle$ is dark, since $H|-\rangle=0$. This is true as long as the probe and driving fields are in two-photon resonance with the transition $|b\rangle \leftrightarrow|c\rangle$. If all the atoms in the medium are in the dark state the medium is transparent to both fields [see probe spectrum in Fig. 2 (dotted line)]. The theoretical transmission spectra are produced by using a density-matrix formalism, and calculating the propagation of a weak probe field.

\section{B. Double-dark resonances}

The usual mechanism by which perturbing the dark state destroys the transparency has been reviewed recently [15]. Here we show that a coherent perturbation, e.g., a weak monochromatic electromagnetic field with Rabi frequency $\Omega_{c}$ coupling an additional state [see Fig.1(b)], can display interesting and highly coherent features. This case is depicted in Fig. 2 (dashed line), where a sharp absorption line appears on resonance, which splits the point of transparency in two. The two transmission maxima are now detuned by
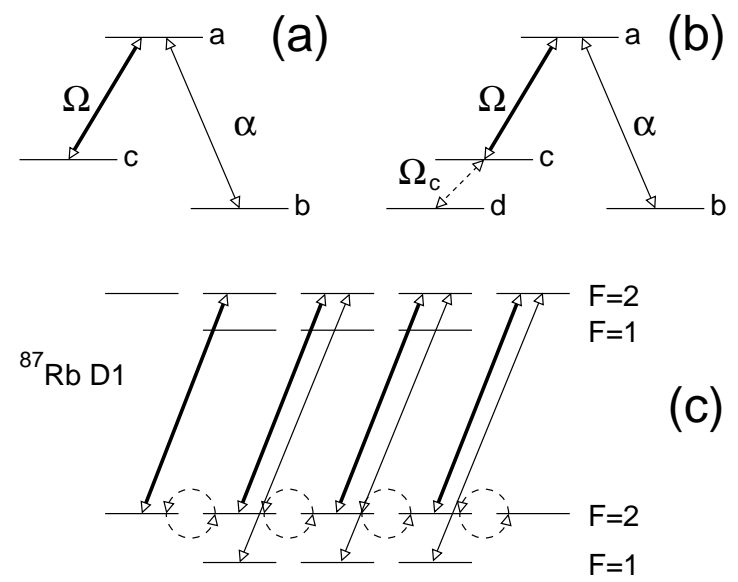

(C)

FIG. 1. (a) Energy-level scheme of a $\Lambda$ system. (b) Extension of (a) by an additional weak coherent field $\Omega_{c}$ to a four-level system. (c) Four-level system in ${ }^{87} \mathrm{Rb}$ as used in the experiment. 


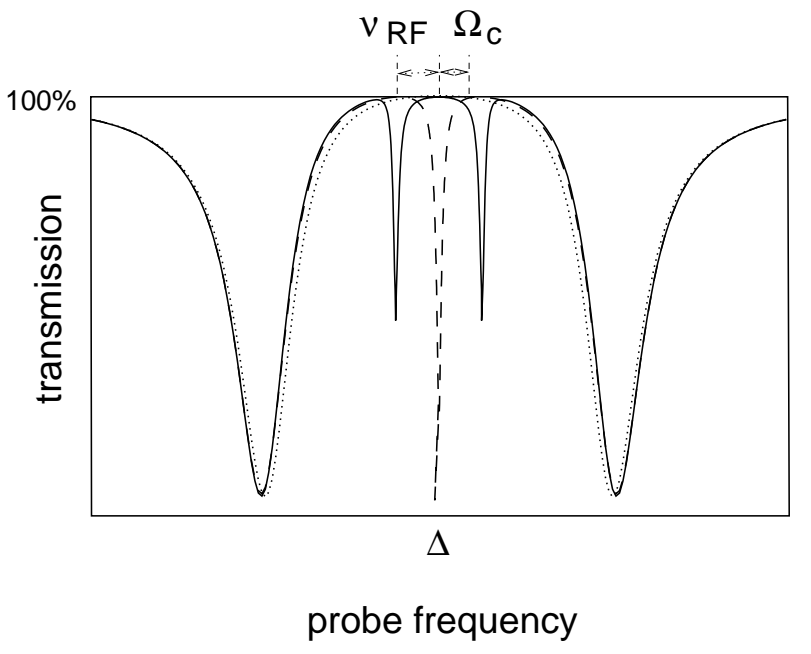

FIG. 2. Transmission spectrum (dotted line) of probe field $\alpha$ of Fig. 1(a) if the drive field $\Omega$ is on resonance. The probe field is resonant at the center of the figure. The dashed line depicts the same transmission spectrum in the presence of an additional weak coherent perturbation [cf. Fig. 1(b)]. The transparency is split by $2 \Omega_{c}$. The solid line is the transmission spectrum in the case treated in this article [cf. Fig. 3(a)], the two additional absorption lines are split by twice the radio frequency $\nu_{\mathrm{RF}}$.

$\pm \Omega_{c}$ compared to resonance. This absorption line in between can be very narrow, as its width scales with the ratio of the intensities $\Omega_{c}^{2} / \Omega^{2}$. Nevertheless, its depth shows that the resonant absorption is as strong as that of the original Stark-split levels. For a simplified explanation of this phenomenon, the dressed-state picture is very useful, where the Hamiltonian is diagonalized with respect to driving and perturbing fields. The resulting resonant state, $|0\rangle \approx|d\rangle$ $+\Omega_{c} / \Omega|a\rangle$, has a small admixture of the excited state, and thus it decays very slowly, which results in a very narrow resonance. In this picture, it is easy to prove that in the allresonant case, detuning the field $\Omega_{c}$ by $\nu_{\mathrm{RF}}$ implies a frequency shift of the novel resonance line by $\nu_{\mathrm{RF}}$.

The experiment described in this paper follows a slightly different setup. The states $|c\rangle$ and $|d\rangle$ are degenerate (i.e., Zeeman sublevels), whereas the new field, a RF field, has the fixed frequency $\nu_{R F}$. This situation can be understood by decomposing the RF field into two effective components that are blue and red detuned by $\pm \nu_{\mathrm{RF}}$ as compared to the transition $|c\rangle \leftrightarrow|d\rangle$. One can thus expect two novel absorption lines rather than one, but with half the absorption depth, shifted by $\pm \nu_{R F}$ compared to the line center, as in Fig. 2 (solid line). In order to understand this behavior, we investigate qualitatively the dressed state system shown in Fig. 3. Specifically, we can gain qualitative insight into the origin of the resulting resonances by consulting the Hamiltonian $\quad H=\hbar \Omega|a\rangle\langle c|+(\hbar / 2) \Omega_{c}\left(\left|d_{1}\right\rangle\left\langle c|+| d_{2}\right\rangle\langle c|\right)$ + H.c. $+\hbar \nu_{\mathrm{RF}}\left(\left|d_{1}\right\rangle\left\langle d_{1}|-| d_{2}\right\rangle\left\langle d_{2}\right|\right)$. Here, $|d\rangle$ is split into $\left|d_{1}\right\rangle$ and $\left|d_{2}\right\rangle$ to include the fact that the RF field is red and blue detuned at the same time. From this Hamiltonian we find the eigenstates and eigenvalues of the dressed-state system

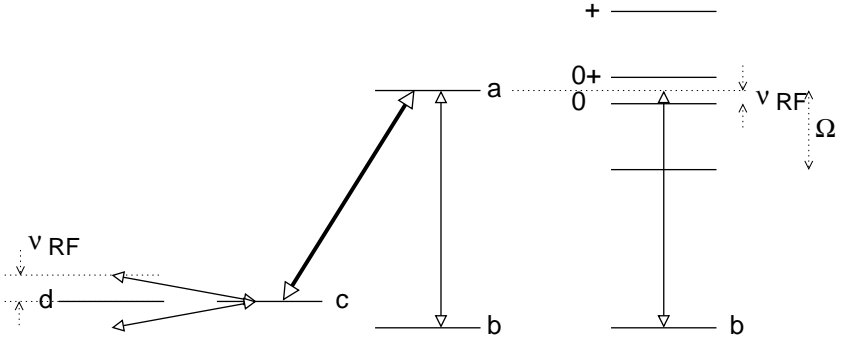

(a)

(b)

FIG. 3. (a) Simple level system of the double dark setup as used in our experiment, where the RF field can be seen as red and blue detuned. (b) Same system, but in a dressed-state picture. The level $|d\rangle$ is split into two by the red and blue detuned RF components, whereas the dressed states $|+\rangle$ and $|-\rangle$ are nearly the same as in a simple $\Lambda$ system.

$$
| \pm\rangle \approx \frac{1}{\sqrt{2}}(|a\rangle \pm|c\rangle), \quad \lambda_{ \pm} \approx \pm \Omega
$$

and

$$
\begin{gathered}
|0 \pm\rangle \approx\left|d_{1 / 2}\right\rangle+\frac{\Omega_{c}}{2 \Omega} f\left(\Omega, \nu_{\mathrm{RF}}\right)\left( \pm|a\rangle+\frac{\nu_{\mathrm{RF}}}{\Omega}|c\rangle,\right. \\
\lambda_{0 \pm} \approx \pm \nu_{\mathrm{RF}} .
\end{gathered}
$$

Here, we use $f\left(\Omega, \nu_{\mathrm{RF}}\right)=\Omega^{2} /\left(\Omega^{2}-\nu_{\mathrm{RF}}^{2}\right)$ and assume $\Omega$ $\neq \nu_{\mathrm{RF}}$. The small admixture of the excited state makes sure that the dressed states $|0 \pm\rangle$, replacing $|d\rangle$, decay with a very narrow linewidth, and the eigenenergies coincide directly with the frequencies of the absorption lines.

\section{Model}

These effects can be quantitatively understood by solving the equations of motion describing the system shown in Fig. 1. The Hamiltonian including probe, drive, and RF fields is given by

$$
\begin{aligned}
H= & \hbar\left(\Omega e^{-i \nu_{\Omega^{t}} t}|a\rangle\left\langle c\left|e^{i \omega_{a c} t}+\alpha e^{-i \nu_{\alpha} t}\right| a\right\rangle\langle b| e^{i \omega_{a b} t}\right. \\
& \left.+\Omega_{c} e^{-i \nu_{\mathrm{RF}^{t}} t}|c\rangle\left\langle d\left|+\Omega_{c} e^{-i \nu_{\mathrm{RF}} t}\right| d\right\rangle\langle c|+\text { H.c. }\right) .
\end{aligned}
$$

Here, we have used the rotating wave approximation (RWA) with regard to probe field (Rabi frequency $\alpha$ ) and driving field (Rabi frequency $\Omega$ ). No RWA is assumed for the RF field. This leads to a von-Neumann equation that has no stationary state, but which can be solved using a Floquet ansatz. The equations leading to the propagation of the weak probe field are for the frequency components of

$$
\rho_{a b}=\sum_{j=-\infty}^{\infty} \rho_{a b}^{(j)} e^{j i \nu_{\mathrm{RF}}}
$$

and the respective series for all the other density-matrix components, we find 


$$
\begin{gathered}
\dot{\rho}_{a b}^{(j)}=-\left(\gamma_{a b}-i \Delta+j i \nu_{\mathrm{RF}}\right) \rho_{a b}^{(j)}+i \alpha \delta_{j 0}+i \Omega \rho_{c b}^{(j)}, \\
\dot{\rho}_{c b}^{(j)}=-\left(\gamma_{c b}-i \Delta+i \Delta_{0}+j i \nu_{\mathrm{RF}}\right) \rho_{c b}^{(j)}+i \Omega \rho_{a b}^{(j)} \\
+i \Omega_{c}\left(\rho_{d b}^{(j+1)}+\rho_{d b}^{(j-1)}\right), \\
\dot{\rho}_{d b}^{(j)}=-\left(\gamma_{d b}-i \Delta+i \Delta_{0}+j i \nu_{\mathrm{RF}}\right) \rho_{d b}^{(j)}+i \Omega_{c}\left(\rho_{c b}^{(j+1)}+\rho_{c b}^{(j-1)}\right),
\end{gathered}
$$

where $\gamma_{a b}, \gamma_{c b}$, and $\gamma_{d b}$ are the coherence decay rates on the respective transitions, and $\Delta=\nu_{\alpha}-\omega_{a b}, \Delta_{0}=\nu_{\Omega}$ $-\omega_{a c}$. Together with the propagation equation for the probe field Rabi frequency

$$
\frac{\partial}{\partial z} \alpha(z, t) \propto \rho_{a b}(t),
$$

the transmission of the field can be calculated and plotted.

First, we are interested only in the main frequency components of the spectrum (i.e., up to $\Omega_{c}^{2}$ ). Assuming that the probe field $\alpha$ is weak and that all population is in state $|b\rangle$, we find for the polarization

$$
\rho_{a b}=i \alpha \frac{\Gamma_{c b} \Gamma_{d b}^{+} \Gamma_{d b}^{-}+\Omega_{c}^{2}\left(\Gamma_{d b}^{+}+\Gamma_{d b}^{-}\right)}{\Gamma_{d b}^{+} \Gamma_{d b}^{-}\left(\Omega^{2}+\Gamma_{a b} \Gamma_{c b}\right)+\Gamma_{a b}\left(\Gamma_{d b}^{+}+\Gamma_{d b}^{-}\right) \Omega_{c}^{2}} .
$$

Here, $\Gamma_{a b}=\gamma_{a b}-i \Delta, \Gamma_{c b}=\gamma_{c b}-i \Delta+i \Delta_{0}$, and $\Gamma_{d b}^{ \pm}=\gamma_{d b}$ $-i \Delta+i \Delta_{0} \pm i \nu_{\mathrm{RF}}$.

Before proceeding we note that while comparing the theoretical predictions to the experimental result, we are interested in the probe field spectrum. But whereas we calculate theoretically exactly the probe transmission, the spectrum analyzer in our experiment measures the beat signal between the (constant) driving and the probe field. Clearly, these quantities are proportional.

\section{Nonlinear sideband generation}

When the RF field becomes sufficiently strong this arrangement also produces efficient nonlinear modulation: sidebands appear in even multiples of the RF frequency $\nu_{\mathrm{RF}}$. This can be explained in the following way: Two RF photons couple (via state $|d\rangle$ ) to virtual states $\left|c_{2 \pm}\right\rangle$, which are lying exactly $2 \nu_{R F}$ above and below the level of $|c\rangle$ (see Fig. 4). The driving field parametrically creates a transparency for the probe field at $2 \nu_{R F}$ above and below its original frequency. Higher-order nonlinearities then can be seen for fourth, sixth, etc., order virtual levels $\left|c_{4 \pm}\right\rangle,\left|c_{6 \pm}\right\rangle$, etc., with decreasing intensities (see Sec. III C).

The spectra for the higher-order sidebands are found by solving Eqs. (4) up to higher orders of $\Omega_{c}$, i.e., fourth, sixth, etc. The results can be read from $\rho_{a b}^{( \pm 2)}, \rho_{a b}^{( \pm 4)}$, etc., and are plotted in Fig. 9.

\section{E. Doppler narrowing}

The experiment is performed in a hot gas and therefore Doppler broadening is an important consideration. We will give here a brief explanation of the impact of Doppler shifts,

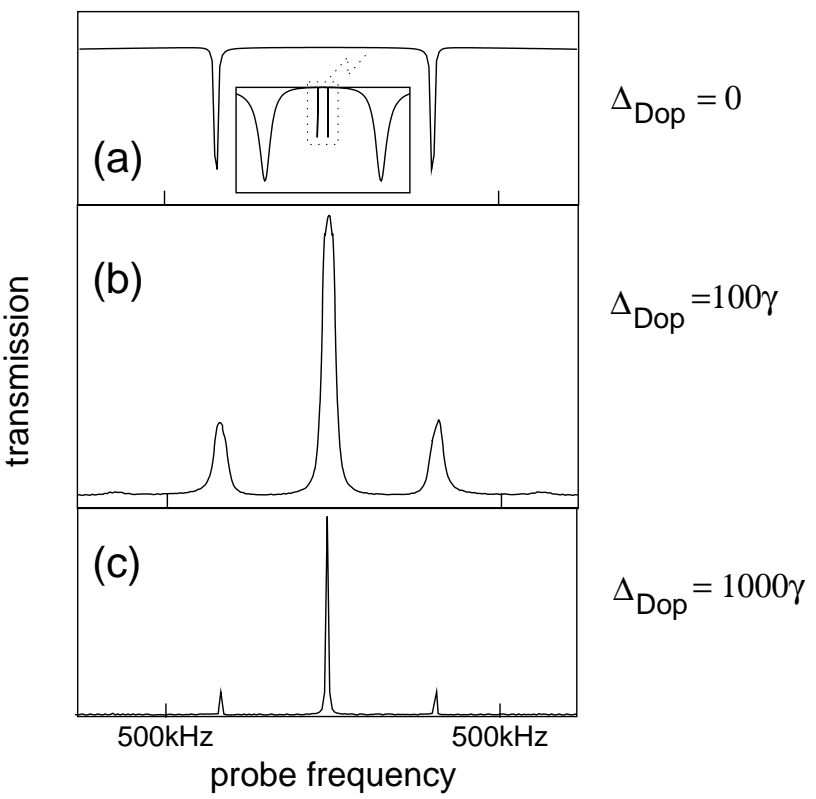

FIG. 4. (a) Transmission spectrum of the probe field, for a situation in which $|c\rangle$ and $|d\rangle$ have the same energy, and the additional field is a fixed-frequency RF field (same as in Fig. 2, solid line, but with parameters similar to those in the experiment). The main figure is a blowup of the inset. (b) Same as (a), but with a (realistic) Doppler broadening of $\Delta_{\text {Dop }} \sim 500 \mathrm{MHz}$, which is approximately 100 natural linewidths of the probe transition. (c) Same as in (b), but with ten times higher Doppler broadening, in order to demonstrate the Doppler narrowing effect.

as they pose an interesting puzzle. For each velocity component of the atomic gas, the probe and drive transitions are affected by Doppler shifts equally, since these shifts depend linearly on the frequency and here the probe and drive fields copropagate. The RF transition, however, is essentially unaffected because of its very low frequency. It can be expected that the transparency on resonance in the Doppler-broadened case gives rise to a transparency whose width decreases for increasing Doppler broadening. Surprisingly, there are Doppler narrowed transmission lines replacing the absorption lines of the Doppler free case. This "Doppler narrowing" can be explained using Fig. 5. For each velocity group there are four eigenvalues in the system, corresponding to Eqs. (1). Each eigenvalue is responsible for an absorption line. In the same limit of small RF field they are

$$
\begin{gathered}
\lambda_{0 \pm}^{\mathrm{Dop}}= \pm \nu_{\mathrm{RF}}, \\
\lambda_{ \pm}^{\text {Dop }}=\frac{\Delta_{\text {Dop }}}{2} \pm \sqrt{\frac{\Delta_{\text {Dop }}^{2}}{4}+\Omega^{2}},
\end{gathered}
$$

where $\Delta_{\text {Dop }}$ is the Doppler frequency shift. Here, we assume that probe and drive fields are on resonance for $\Delta_{\text {Dop }}=0$. The energy eigenvalues $\lambda_{ \pm}^{\text {Dop }}$ and $\lambda_{0 \pm}^{\text {Dop }}$ of the atomic dressed states as functions of the Doppler shift $\Delta_{\text {Dop }}$ are shown in Fig. 5. Eigenvalues $\lambda_{ \pm}^{\text {Dop }}$ (shown in the figure as broken lines) are responsible for the Stark split absorption lines in usual EIT. The dotted lines show all eigenvalues without 


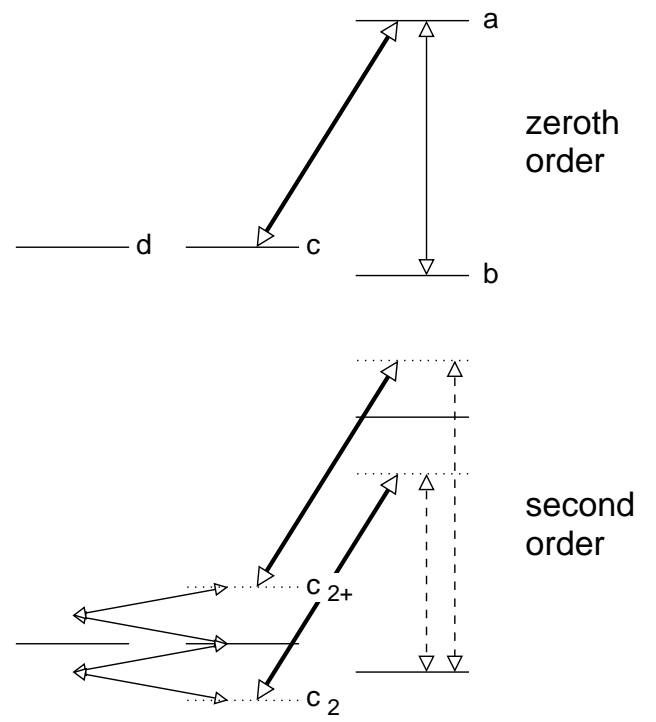

FIG. 5. Level structure illustrating how a nonresonant (i.e., finite frequency) RF field can generate second-order frequencies. The solid levels are pure atomic states, the dashed ones are virtual states created by the fields. The solid field arrows designate ingoing and outgoing fields, whereupon the dashed arrows signify fields that are created parametrically, and thus only outgoing.

both, driving and RF fields, $\Omega=\Omega_{c}=0$. (Note that in this case there are no avoided crossings.) With both fields present all anticrossings appear in Fig. 5 (solid lines). Performing the Doppler average over all components (which, in mathematical terms, means a Gaussian average over all $\Delta_{\text {Dop }}$ ) leads to an absorption profile where narrow transparency lines can be seen very close to the $\pm \nu_{\mathrm{RF}}$ eigenvalues. The reason is obvious from Fig. 5, where the regions very close to $\pm \nu_{\mathrm{RF}}$ and to 0 are the only ones that are not crossed by any of the

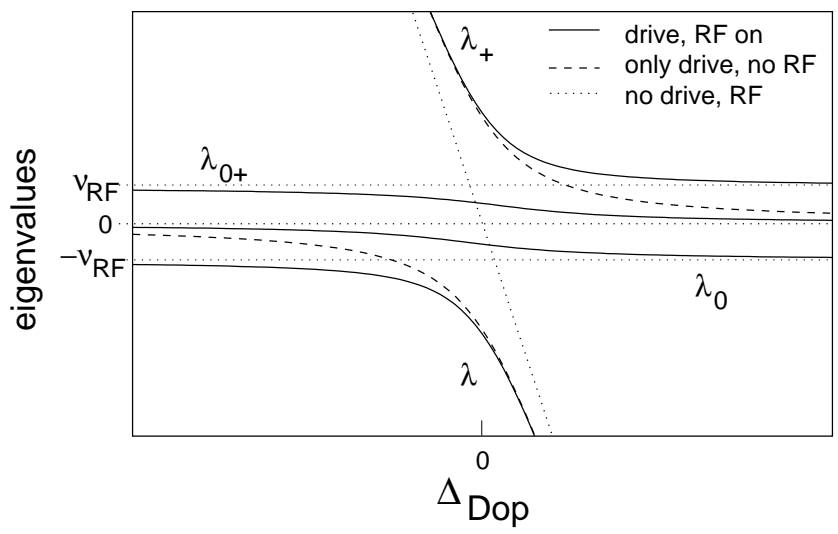

FIG. 6. Eigenvalues of the system as a function of the Doppler shift $\Delta_{\text {Dop }}$, i.e., for all different velocity groups. The eigenvalues in this figure depict the true energy level of the atomic states, and therefore would be at the center of any absorption line, whereas transmission would be visible somewhere between the eigenvalues. Dotted lines depict the energy levels of the atomic states when neither the drive $\Omega$ nor the RF field $\Omega_{c}$ is present. The broken lines are for $\Omega_{c}=0$, but $\Omega \neq 0$. The solid lines give the solution for all three fields, RF, drive, and probe, present.

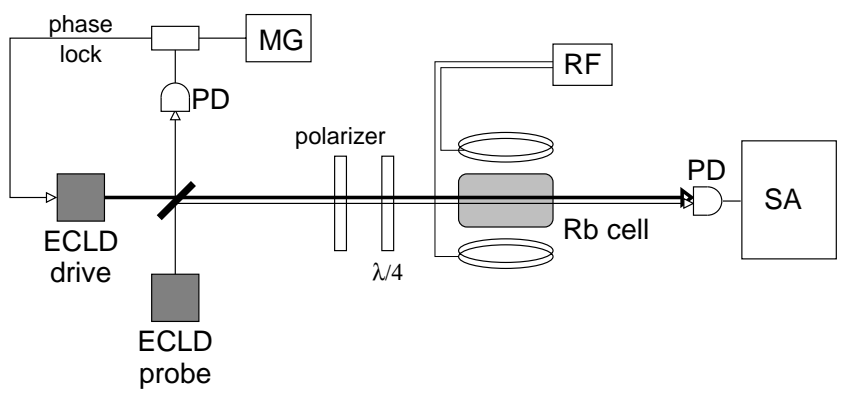

FIG. 7. Experimental setup. The drive and probe fields are produced by phase-locked extended cavity laser diodes (ECLD) and $\sigma_{+}$-polarized by a polarizer and $\lambda / 4$-wave plate. A spectrum analyzer (SA) examines the probe-drive beat note. The weak-coupling field is produced by a radio frequency $(\mathrm{RF})$ coil.

eigenvalues over the width of all $\Delta_{\text {Dop }}$. Thus these lines are nearly at the frequency where there is maximum absorption in the Doppler free case, and become narrower the broader the Doppler shift is spread.

The resulting transmission curve can be seen in the simulation of Fig. 6, where the three lines (one EIT-close to 0 - and two double dark lines-close to $\left.\pm \nu_{\mathrm{RF}}\right)$ are clearly visible. The parameters in (b) are chosen to coincide with the experimental reality: The Doppler broadening in our experiment is $530 \mathrm{MHz}$.

The sideband generation is much more pronounced in the Doppler-broadened medium than in the nonbroadened one, which seems surprising at first. It helps to remember that the RF novel lines are absorption resonances in the nonbroadened case, whereas they are transmission resonances in the Doppler-broadened case. These transmission lines coincide with the zero-order dark resonance lines of the sideband spectra (see, e.g., Fig. 9). Consequently, the ratio of neighboring sideband intensities $I_{4 \nu_{\mathrm{RF}}} / I_{2 \nu_{\mathrm{RF}}} \approx I_{2 \nu_{\mathrm{RF}}} / I_{0}$ is roughly

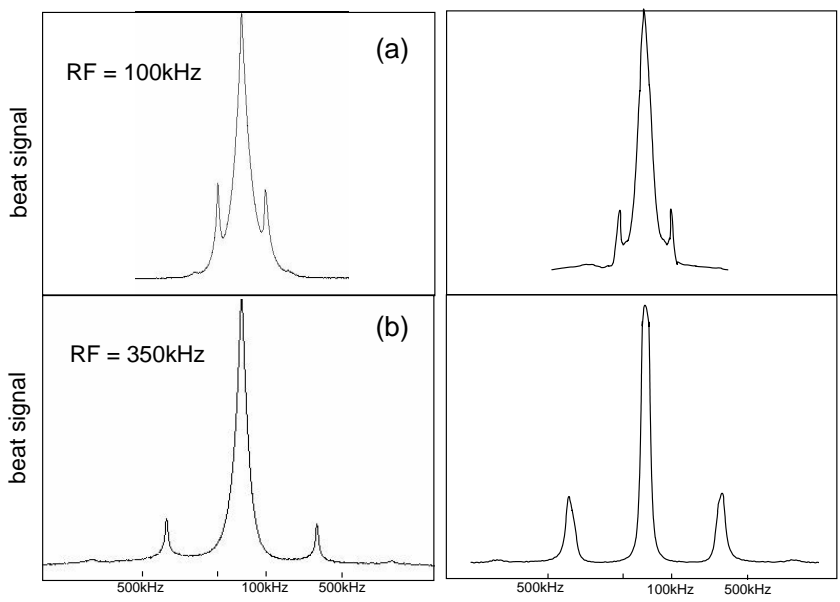

FIG. 8. Transmission curves for two different RF frequencies, experiment (right) and theory (left). In (a) the RF is $100 \mathrm{kHz}$, where the new resonances still overlap with the dark resonance peak, whereas in (b), with a RF of $350 \mathrm{kHz}$, these lines are well resolved. The RF magnetic-field component in these curves was $20 \mathrm{mG}$ in (a) and $80 \mathrm{mG}$ in (b). 

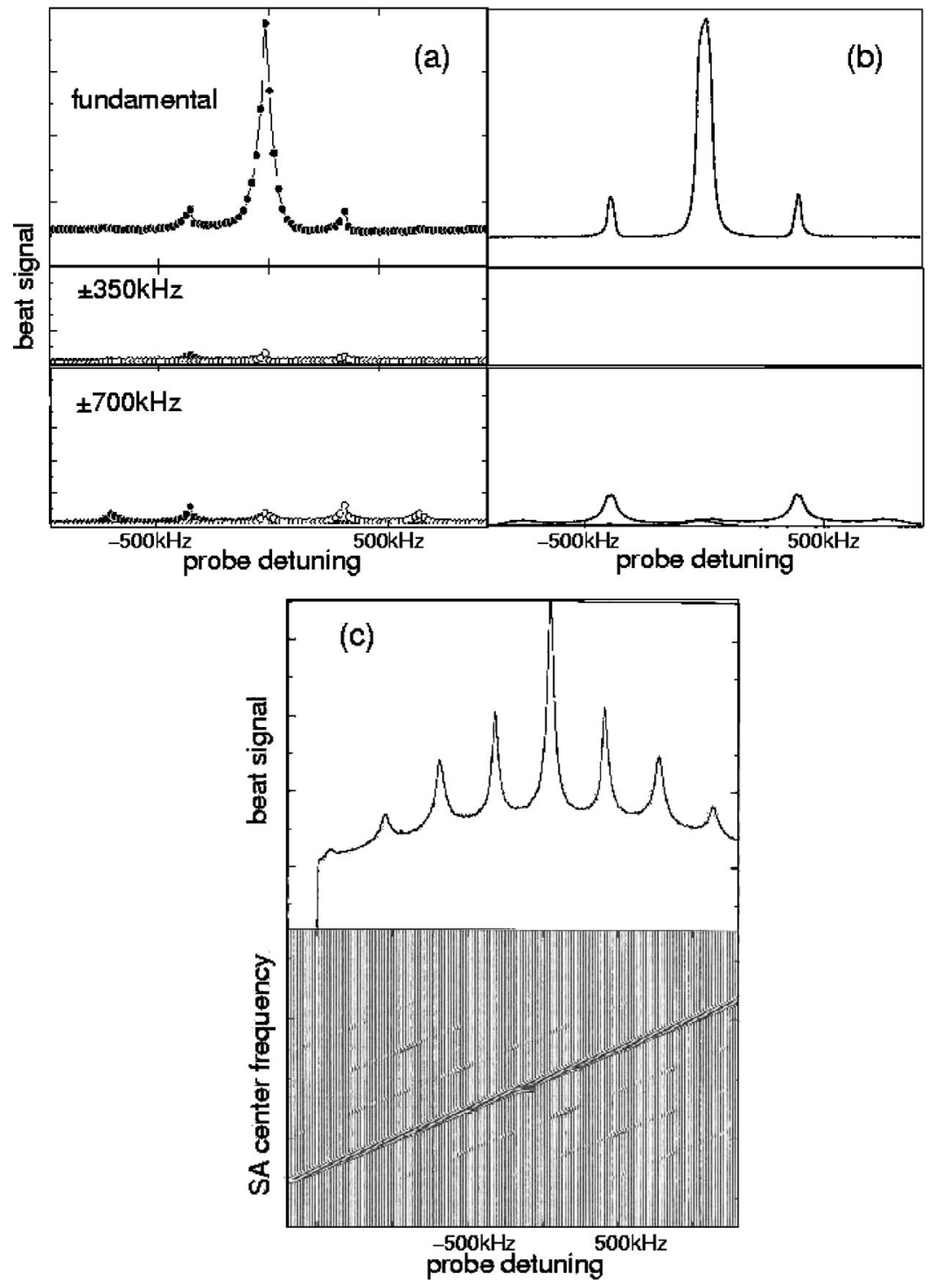

two orders of magnitude higher for Doppler-broadened media, such that the three-peak structure of the fundamental mode can also be seen in the second-order sidebands [Fig. $9(\mathrm{a}),(\mathrm{b})]$.

\section{EXPERIMENT}

\section{A. Setup}

The experiment was performed on the $\mathrm{D}_{1}$ resonance line of ${ }^{87} \mathrm{Rb}$ (nuclear spin $I=3 / 2$, wavelength $795 \mathrm{~nm}$ ). The experimental setup is shown in Fig. 7. In our experiment, external cavity diode lasers were used for both the drive and the probe fields. The two collinear beams, both of which are circularly polarized and which have the same diameter of 0.1 $\mathrm{cm}$, are overlapped and directed into a glass cell of length $l$ $=4 \mathrm{~cm}$, filled with rubidium vapor. The vapor consisted of $4 \times 10^{11} \mathrm{~cm}^{-3}\left(T=65^{\circ} \mathrm{C}\right)$ of the natural abundance of ${ }^{85} \mathrm{Rb}$ and ${ }^{87} \mathrm{Rb}$, plus 3 Torr of Ne buffer gas. The buffer gas is needed in order to reduce the relaxation rate of the groundstate coherence, which for vapor densities of this order stems mostly from the $\mathrm{Rb}$ atoms entering into and leaving the in-
FIG. 9. (a) Experimental spectra of the beat note between the transmitted drive and probe fields. In the upper picture, the spectrum analyzer (SA) is centered around the fundamental, i.e., resonance frequency. In the two lower graphs, the SA is tuned to the firstand second-order sidebands, respectively. It is clear from these curves that only even order sidebands are supported. (b) Simulations of curves in (a). Note that while in theory there are no firstorder sidebands, there are small ones in the experimental data. (The reason for that is a broad fundamental mode.) (c) The upper curve is similar to the one in (a), but with much stronger RF fields (160 mG) such that multiple sidebands are visible. This spectrum is an integration over all SA frequency curves, which are depicted explicitly in the lower picture. teraction region defined by the laser beams. Thus the groundstate decoherence rate depends on the diameter of the laser beams and in our case can be estimated to be of the order of $5 \mathrm{kHz}$. The cell was mounted inside a three-layered magnetic shield. Perpendicular to the laser beams the RF field could be applied, using transverse coils. The cell was heated using DC current in a high-resistance wire wound on the outside of the middle shield. The atomic density can then be estimated from the temperature in the cell using well-known vaporpressure curves, e.g., from Ref. [17]. The drive laser was tuned to the $5 S_{1 / 2}(F=2) \leftrightarrow 5 P_{1 / 2}(F=2)$ transition; the probe laser was tuned to the $5 S_{1 / 2}(F=1) \leftrightarrow 5 P_{1 / 2}(F=2)$ transition. This configuration of drive and probe fields creates an appropriate $\Lambda$ system within the ${ }^{87} \mathrm{Rb} D_{1}$ manifold [see Fig. 1(c)].

There must be minimal relative laser jitter between probe and drive fields, so the probe laser was phase-locked to the driving field frequency with an offset frequency of 6.835 $\mathrm{GHz}$, which is equal to the hyperfine ground-state splitting between the $5 S_{1 / 2}(F=1)$ and $5 S_{1 / 2}(F=2)$ states. This offset is fixed by a tunable microwave frequency synthesizer. After 
passing through the cell, the laser beams impinge on a fast photodiode. The beat signal from this photodiode is monitored by a microwave spectrum analyzer, which is used as a broadband filter amplifier with a bandwidth of $3 \mathrm{MHz}$, centered at the hyperfine splitting frequency.

\section{B. Spectrum}

The line shape of the dark resonances are determined by scanning the probe laser frequency, which is accomplished by tuning the frequency of the synthesizer. While the laser power of the driving field can be changed from 0 to $6 \mathrm{~mW}$, the probe laser is held at $1 \%$ of the drive power. With these parameters a broadening of the dark resonances could be observed with a slope of $30 \mathrm{kHz} / \mathrm{mW}$. Field broadening and density narrowing of the dark resonance were investigated in Ref. [18]. In order to observe EIT resonance and pronounced double dark structure, the driving field has to be strong enough to make the additional double dark structures lie well within the EIT resonance. (This was tested experimentally when the drive laser power is low, the novel features are not detectable.) The optimal working point thus was determined to be $2.2 \mathrm{~mW}$ for the drive laser.

The double dark resonance structures can be seen in Fig. 8(a). These additional resonances are induced by the RF field at a frequency of $100 \mathrm{kHz}$. The additional resonances in Fig. 8(b) are created by the RF field at $350 \mathrm{kHz}$. The new transparency lines are well outside the EIT peak. As can be seen in the figures, the frequency interval between the dark resonance and the new resonances indeed equals the RF frequency. The width of the novel resonances is $20 \mathrm{kHz}$, which suggests a total decoherence rate between the three ground states $|b\rangle,|c\rangle$, and $|d\rangle$ of $20 \mathrm{kHz}$.

\section{Sideband generation}

The spectral distribution of the transmitted laser light was also studied. For this purpose the spectral analyzer was switched to a narrow band regime (bandwidth $30 \mathrm{kHz}$ ) and the central frequency of the bandwidth was scanned. The laser frequency during the spectrum analyzer scan was fixed. In this procedure several new optical components (sidebands) can be observed, which are separated by frequency intervals equal to harmonics of the applied magnetic field frequency. Obviously these optical components are generated during the propagation of laser light through the coherently prepared $\mathrm{Rb}$ vapor. In order to record their dependence on the laser frequency, we present snapshots where frequency steps of $20 \mathrm{kHz}$ are taken, see Fig. 9. The fundamental frequency here is the frequency of the beat note of the driveand probe-laser beams without the cell. The signal at the fundamental frequency is similar to the broadband detected signal in Fig. 8(b), as could be expected. One can see the different behavior of the odd and even harmonics (sidebands). The amplitude of the second-order sidebands that are observed at $\pm 700 \mathrm{kHz}$ is higher than the the amplitude of the first-order sidebands that are observed at $\pm 350 \mathrm{kHz}$. The reason that they are seen at all stems from the fact that the fundamental mode is broad enough to seep into the firstorder sidebands. In this case, the virtual states $\left|d_{ \pm}\right\rangle$, detuned by $\pm \nu_{\mathrm{RF}}$ from state $|d\rangle$, are directly coupled to $|a\rangle$, which leads to first-order sidebands. Note that in the simulations (Fig. 6) no first-order sidebands are present.

The three-peak structure in the main diagonal of the lower curves of Fig. 9(c) is the main contribution to the integrated spectrum (upper curve), and for low RF intensities is all that is seen [e.g., in (a),(b)]. In reality, however, this contribution makes up only the fundamental mode. Sidebands originate in the resonances with $\left|c_{2 \pm}\right\rangle,\left|c_{4 \pm}\right\rangle$, etc., and can be seen for stronger RF intensity, such as in Fig. 9(c). In the lower picture, each of the three diagonal absorption maxima, which are offset from their neighbors by $\pm 2 \nu_{\mathrm{RF}}$, consists of the fundamental three-peak structure. Finally, in the integrated picture, they add up to the multipeak spectrum in the upper curve.

\section{CONCLUSION}

In conclusion, we have experimentally demonstrated novel coherent resonances as predicted in Ref. [15]. These novel resonances are very sharp and highly sensitive to frequency and intensity of a RF field, which perturb the dark resonance. In addition, because of higher-order coupling, efficient nonlinear sideband generation was demonstrated. This scheme can be used in applications like sensitive nonlinear measurements such as Ref. [19] and UV laser generation [20].

We want to thank M.O. Scully for his interest in this project and discussions leading to it, and we want to thank the Office of Naval Research and the National Science Foundation for support. S.F.Y. also acknowledges the Humboldt Foundation for financial support.
[1] S. Harris, Phys. Today 50(7), 36 (1997), for review.

[2] M.O. Scully and M.S. Zubairy, Quantum Optics (Cambridge University Press, Cambridge, 1997), for references.

[3] A.J. Merriam, S.J. Sharpe, M. Shverdin, D. Manuszak, G.Y. Yin, and S.E. Harris, Phys. Rev. Lett. 84, 5308 (2000).

[4] M.D. Lukin and A. Imamoğlu, Phys. Rev. Lett. 84, 1419 (2000).

[5] P.R. Hemmer, D.P. Katz, J. Donoghue, M. Cronin-Golomb, M.S. Shahriar, and P. Kumar, Opt. Lett. 20, 982 (1995).
[6] L.V. Hau, S.E. Harris, Z. Dutton, and C.H. Behroozi, Nature (London) 397, 594 (1999).

[7] M.M. Kash, V.A. Sautenkov, A.S. Zibrov, L. Hollberg, G.R. Welch, M.D. Lukin, Y. Rostovtsev, E.S. Fry, and M.O. Scully, Phys. Rev. Lett. 82, 5229 (1999).

[8] D. Budker, D.F. Kimball, S.M. Rochester, and V.V. Yashchuk, Phys. Rev. Lett. 83, 1767 (1999).

[9] O. Kocharovskaya, Y. Rostovtsev, and M.O. Scully, Phys. Rev. Lett. 86, 628 (2001). 
[10] C. Liu, Z. Dutton, C.H. Behroozi, and L.V. Hau, Nature (London) 409, 490 (2001).

[11] D. Phillips, A. Fleischhauer, A. Mair, R. Walsworth, and M.D. Lukin, Phys. Rev. Lett. 86, 783 (2001).

[12] R.L. Walsworth, S.F. Yelin, and M.D. Lukin, Opt. Photonics News 15, 50 (2002), and references therein.

[13] S.E. Harris and A.V. Sokolov, Phys. Rev. Lett. 81, 2894 (1998).

[14] K. Hakuta, L. Marmet, and B.P. Stoicheff, Phys. Rev. A 45, 5152 (1992).

[15] M.D. Lukin, S.F. Yelin, M. Fleischhauer, and M.O. Scully,
Phys. Rev. A 60, 3225 (1999).

[16] C.Y. Ye, A.S. Zibrov, Y.V. Rostovtsev, and M.O. Scully, Phys. Rev. A 65, 043805 (2002).

[17] A.N. Nemeyanov, Vapor Pressure of Chemical Elements (Elsevier, Amsterdam, 1963).

[18] V.A. Sautenkov, M.M. Kash, V.L. Velichansky, and G.R. Welch, Laser Phys. 9, 889 (1999).

[19] S.F. Yelin and P.R. Hemmer, Phys. Rev. A 66, 013803 (2002).

[20] E.S. Fry, M.D. Lukin, T. Walther, and G.R. Welch, Opt. Commun. 179, 499 (2000). 